\title{
Comparative Study of Speed Protocols in Wireless Sensor Network
}

\author{
Harshkumar Thakar \\ M.E.I.T (pursuing) \\ Thakur College of Engineering \\ And Technology \\ University of Mumbai
}

\author{
Sangeeta Vhatkar \\ Assistant professor, TCET \\ Ph.D. Scholar \\ P.I.E.T, Nagpur
}

\author{
Mohommad Atique \\ Associate Professor \\ P.G. Department of Computer \\ Science S.G.B.A.U, \\ Amravati
}

\begin{abstract}
Wireless sensor network (WSN) [1] is based on distributed event-based system that is different from the traditional communication network. The main goal of the network is to deliver a data packet with the highest efficiency and quality of service. In WSN, Quality of service (QoS) [2] is an important performance indicator, therefore the QoS routing protocols are designed to provide more efficiency and quality of service. Here they concentrate on SPEED [3] protocol (Stateless Protocol for End to End Delay) is a QoS based protocol and which provides efficiency, softness-real time communication, desired delivery speed and a low overhead over the network. MMSPEED [4] is another multi-path multi SPEED protocol, which provides more efficiency and minimal control overhead over the network. This paper represents SPEED protocol and a comparative study on several versions of SPEED protocols.
\end{abstract}

\section{General Terms}

Wireless sensor networks, Routing Protocol, Quality of Service, Wireless Sensor Network.

\section{Keywords}

WSN, QoS Routing, SPEED, MMSPEED, FT-SPEED, EESPEED.

\section{INTRODUCTION}

A wireless sensor network (WSN) [1] is a gathering of particular transducers with a correspondences base for observing and recording conditions at various locations. WSN monitored parameters are wind direction and speed, humidity, light intensity, vibration intensity, sound intensity, temperature, force per unit area, power-line potential, chemical concentrations and pollutant levels.

A wsn comprises of various identification stations called sensor hubs, every node is little, lightweight and compact. In a network, sensor node is made of microcomputer, transceiver transducer, and a power source. The transducer delivered electrical signals which are taking into sensed physical impacts and phenomena. The microcomputer procedures a sensor node and stores the output. The transceiver gets commands from a central information processing system and transmits data to that computer. The capacity of every sensor node is gotten from a battery.

In the remote sensor organize there are numerous routing methods to send a bundle source to destination, in which they are Essentially separated into Quality of Service routing (Qos),

Hierarchical routing and location based routing protocol. These conventions are further isolated into numerous different conventions, in this paper, they concentrate on QoS routing protocol, SPEED routing protocol and SPEED based routing protocol.

\section{QUALITY OF SERVICE ROUTING PROTOCOL}

However, a wireless sensor network is resource constrained and poses many challenges while designing an efficient routing protocol for deadline-driven traffic. Due to the limited battery power of the sensor nodes, it is important that the routing is energy efficient, in order to increase in the lifetime of the network.

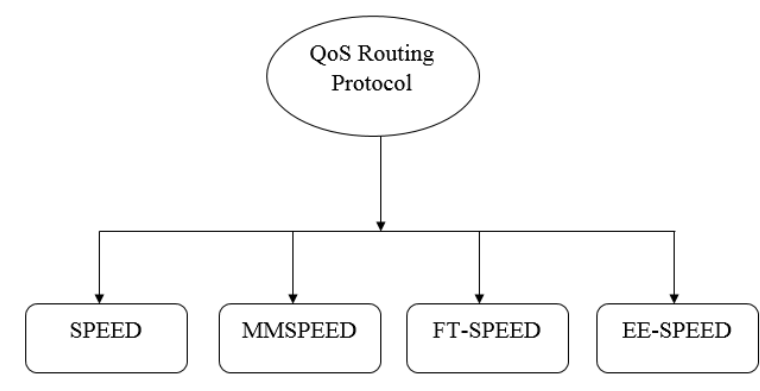

Fig 1: QoS Routing Protocol

In expansion to energy requirements, every sensor node has the low transforming capacity, lower memory power, and constrained transmission energy. As a consequence, these constraints impose an all-important prerequisite on any QoS support mechanisms in WSNs: simplicity. In most WSN applications, traffic mainly flows from a large number of sensor nodes to a small subset of sink nodes. QoS mechanisms should be designed for unbalanced QoSconstrained traffic.

\section{SPEED ROUTING PROTOCOL}

In QoS SPEED [3] routing protocol is designed to provide soft-real time communication for maintaining desire delivery speed across the networks to minimize end to end delay. In this protocol each node has only information about its neighbor and geographic location information to make localized routing decisions. So that this protocol is called as "stateless", as it doesn't utilize directing tables, accordingly it expend insignificant memory use.

SPEED utilized the geographic area to make localized routing choices. The refinement is that SPEED protocol is intended to give a soft real-time communication service and handle blockage, which are not the fundamental objectives of past Quality of Service based routing protocols. SPEED protocol gives a blend of network layer and MAC layer adaptation that 
adequately manages long term congestion issues. For the best of understanding, no routing algorithm has been particularly planned to give soft real-time guarantees for sensor networks.

\subsection{Design Goals}

Outline is motivated by the perception that vary, then wired network, where delay is free of the physical separation between the source and destination, in remote sensor organizes, the end-to-end deferral relies on upon single hop delay, as well as similarly on the distance a packet travels.

Speed protocol gives three sorts of real time communication strategy in particular, real-time area-anycast, real-time unicast, and real-time area-multicast, for WSN. And the speed protocol has taking after outline objectives like, Stateless Architecture, Soft Real-Time, Minimum MAC Layer Support, QoS Routing and Congestion Management, Traffic Load Balancing, Localized Behavior, Void Avoidance.

\subsection{SPEED Architecture}

SPEED protocol keeps up a desired delivery speed across sensor networks arranges by both diverting activity at the networking layer and generally managing packets sent to the MAC layer. It comprises of the accompanying segments are An API, A neighbor beacon exchange scheme, A delay estimation scheme, The SNGF (Stateless Non-deterministic Geographic Forwarding algorithm), A Neighborhood Feedback Loop (NFL), Backpressure Rerouting and Last mile processing.

SNGF is the directing module in charge of selecting the next hop applicant that can confirm the desired delivery speed. At the point when clogging happens the Backpressure routing and NFL are two modules to scale down or redirect traffic, so that SNGF has open plausibility to look over. The last mile methodology is offered to hold the three communication semantics. Delay estimation is the component by which a node picks whether or not blockage has come. Furthermore, and beacon exchange provides the geographic arranging of the neighbor node so that SNGF can able to perform geographic based routing

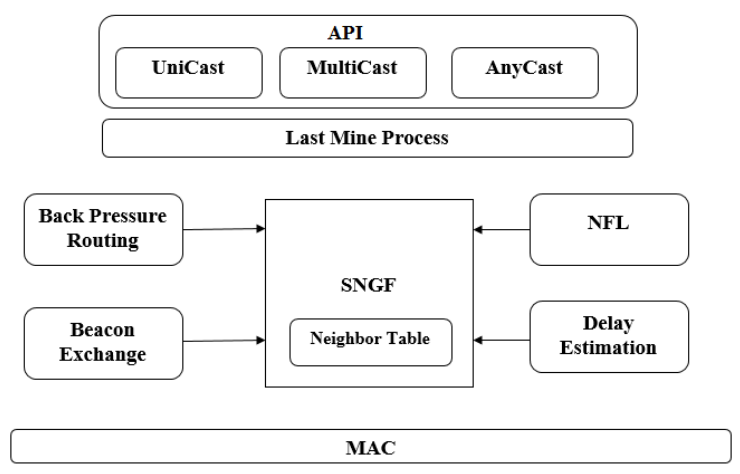

Fig 2: SPEED Architecture

Numerous brilliant protocols have been created for sensor networks. On the other hand, sensor systems have extra essentials that were not particularly coordinated. These include real-time requirements and clients which are seriously constrained in computing power, data transfer capacity, and storage. SPEED keeps up a desired delivery speed over the network through a novel mixture of feedback control and nondeterministic QoS-aware geographic forwarding. This blend of the MAC and network layer adjustment enhances the endto-end delay and gives great reaction to congestion and voids.

\section{SPEED BASED ROUTING PROTOCOL}

In the below section they are examining the SPEED based routing protocol like MMSPEED Protocol, FT-SPEED Protocol and EE-SPEED Protocol.

\subsection{MMSPEED Routing Protocol}

The Multipath and Multi-SPEED Routing (MMSPEED) [4] protocol is an extension of the SPEED protocol. It is planned to provide probabilistic QoS differentiation with regard to timeliness and reliability domains, which spans over network layer and medium access control (MAC) layer. The significant objective is to give QoS separation in two quality areas, namely, reliability and timeliness, so that packets can pick the most legitimate combination of service alternatives relying upon their timeliness and reliability demands. For the timely delivery of packets, MMSPEED gives a many delivery speed options for every incoming packet. Each incoming packet is placed in appropriate queues according to its speed class. After this the packets in the greatest speed queue are served on the premise of the FCFS, trailed by the following highest speed queue and so on. Some other significant property of MMSPEED is an end-to-end QoS provisioning with neighborhood choices at every intermediate node without end-to-end path revelation and support. This trait is important for scalability to extensive sensor networks, network dynamics self-adaptability to, and suitability to both aperiodic and periodic traffic flows.

\subsubsection{MMSPEED Architecture}

The MMSPEED routing protocol is arranged with two critical objectives are localized packet routing without global network state update and a priori path setup, to giving separated QoS choices in timeliness and reliability fields. For the restricted packet routing without end-to-end path delivery and support, they grasp the geographic directing instrument based on location awareness. Foremost, they assume that the packet's destination is characterized by a geographic location as opposed to node ID as justified in the SPEED protocol. Moreover, every sensor node is taken up to be aware of geographical location using GPS or distributed location service.

The range information can be exchanged with brief neighbors with "discontinuous area upgrade packets." Therefore, every node is aware of its quick neighbors inside its radio extent and their regions. Utilizing the neighbor areas, every node can mainly settle on every packet routing decision such that packets progress geographically towards their last destinations. In the event that every last node exchanges the packet to a neighbor closer to the objective range, the packet can inevitably to be conveyed to the destination without global topology data. The localized geographic routing has the accompanying three focal points in sensor systems: Adaptability to a huge and dense sensor network. 

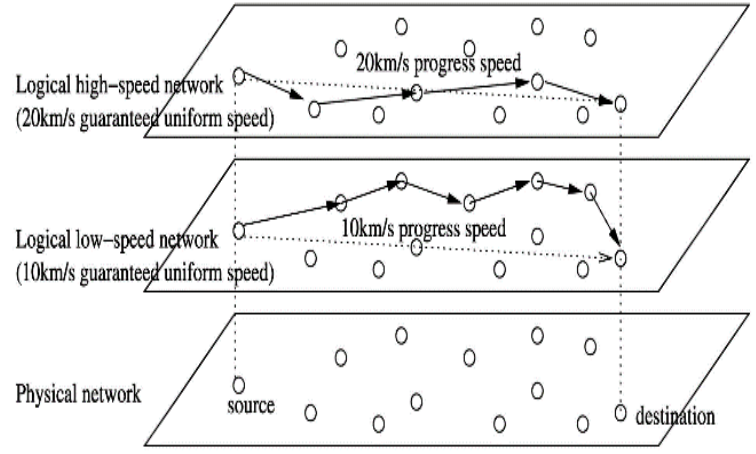

Fig 3: MMSPEED Architecture [4]

No path setup and recovery response time-suitable for both discriminating aperiodic and periodic packets.

Every packet path disclosure bringing about self-adjustment to network elements. Their objective is to give ensured packet delivery benefits in both auspiciousness and dependability domain while saving the advantages of localized geographic routing.

MMSPEED provide service differentiation and probabilistic QoS ensures in the reliability and timeliness domains. For the timeliness area, they give different network-wide speed options so that different traffic types can dynamically pick the best possible speed alternatives for their packet relying upon their end-to-end deadlines. For the dependability domain, they utilize probabilistic multipath forwarding to control the amount of packet delivery paths relying upon the obliged endto-end reaching probability. These systems are actualized in a localized manner with dynamic remuneration to compensate for the mistakes of local choices as packets advancement towards their destinations. Since the proposed instruments work locally at every node without global network state data and end-to-end path setup, it can spare charming properties, for example, scalability for large sensor networks, to network dynamics, self-adaptability and propriety for earnest aperiodic and periodic packets. As a result, MMSPEED can altogether enhance the viable limit of a sensor arrange regarding number of streams meeting both reliability and timeliness requirements.

\subsection{FT-SPEED}

The protocols which are clarified in the past segments is give soft-real time communication and decreased the overhead to provide efficiency in the network, however the void issue hasn't been taken care of well in current routing protocols. Thus they introduce a fault-tolerant real-time routing protocol FT-SPEED. In FTSPEED [5], void announces scheme is centered to keep the packets making the void through one path to the routing path. To send the packet around two sides of the void to ensure that packet to be delivered instead of just being dropped, FT-SPEED presents the void bypass scheme.

\subsubsection{Problem Definition}

At the point when a package hits a node that causes a void Destination set, it is affirmed to have turned over a stuck node. SPEED performs rerouting around a void in the same way as it handles congestion. The figure 4(A) shows of the void avoidance scheme. Similarly we can see, node (b) is a stack node as it causes an empty FS (target). At the point when the packets touch base at the node $b$, it will send $a$ backpressure beacon with its ID (Identification), AvgSendToDelay $=\infty$ and the Destination.

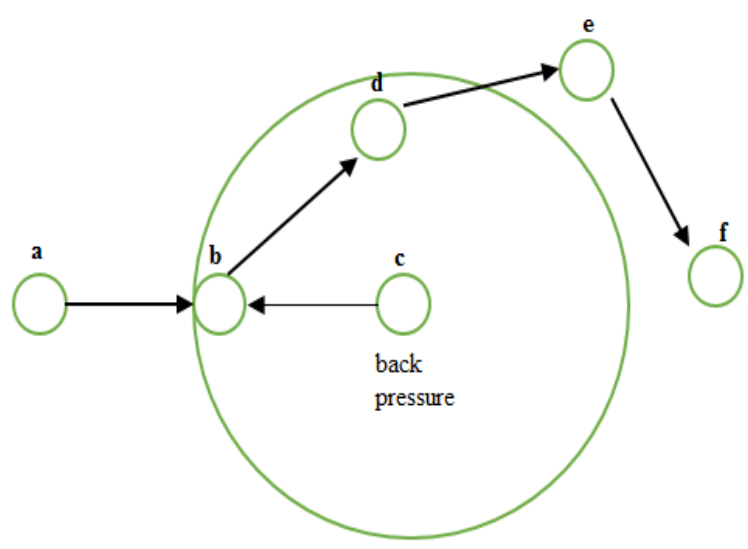

Fig 4 (A): SPEED Void Avoidance Scheme

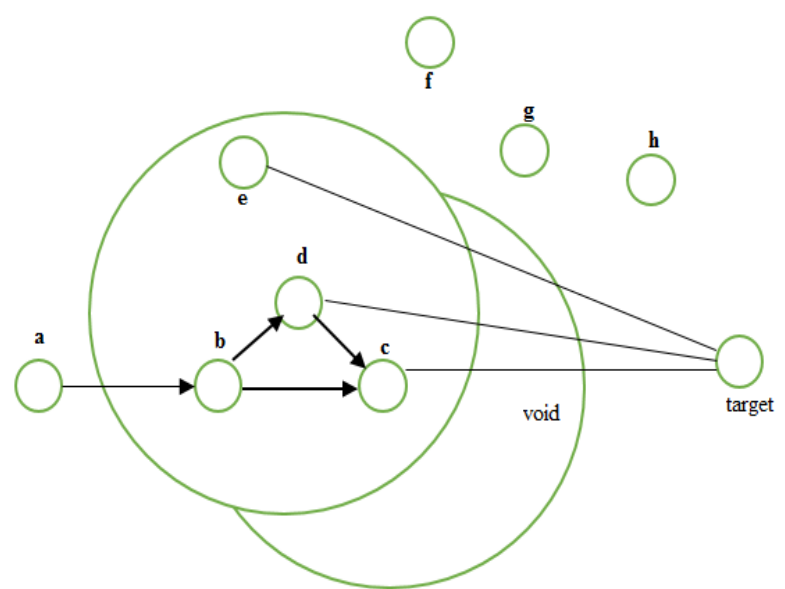

Fig 4 (B): FT-SPEED Void Avoidance Scheme

Node a will set the SendToDelay for node $b$ to $\infty$ and quit transmitting packets to node b If node c doesn't exist, further backpressure will happen until another course is discovered However, consider the accompanying circumstance as demonstrated in Figure 4(B) when node a gets the backpressure beacon form node b, SNGF in SPEED will pick another node which fulfilled the speed necessity in FS as its next hop. In this situation, expected node $\mathrm{c}$ is in the node a's FS set, and node c's just forwarding neighbor is node $b$. At the point When node $\mathrm{c}$ receives the packet from node $\mathrm{a}$, it has no option but to transport the packet to node $b$. As portrayed over, all packets received by node $b$ will be dropped. However, node (a) has no idea about this. It will continue sending the received packets to node $\mathrm{c}$, and these packages are missed by the node $b$ at the nearby. The packets ought not to be conveyed to the nodes which will lead them to the stuck node if another way exists.

\section{FT-SPEED: Fault Tolerance and Real Time Protocol}

Here they proposed two strategy to remove the void from the network.

\section{- Void Announce Scheme}

On the off chance that the node send packet to the stuck node through different path, they first characterize the network void range node and void edge node A void announce scheme is 
basically proposed to dodge the packets be conveyed the nodes which has stuck node as forwarding neighbor. The arrangement of a forwarding neighbor set table utilized as a part of the plan is indicated as Figure (5) at the point when a node gets a packet for the objective, it will check the sending set to that destination. In the event that the sending set is invalid, the node will know itself is a stuck node and telecast a backpressure beacon with its ID, the AvgSendToDelay $=\infty$, and Destination to its neighbor nodes. All nodes keep this node as their sending neighbor will set the SendToDelay for adhered node to $\infty$ when get that beacon. Be that as it may, the ActualDelay value in FS table shouldn't be changed. ActualDelay records the postponement of the real packet delivery delay between nodes. If the neighbor node is not a void node, the ActualDelay is equivalent to the SendToDelay

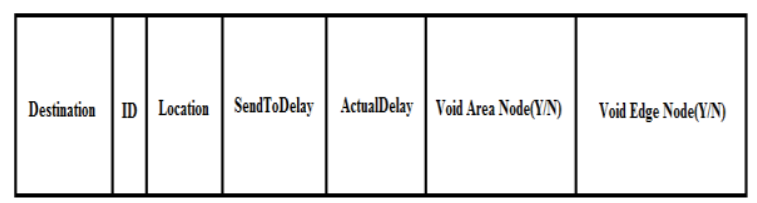

Fig 5: Neighbor Set Table

They are figured by the Delay Estimator which portrayed certainly in SPEED. Nodes redirect packets to void area past will quit sending and pick other sending node as its next hop if exist. On the off chance the node discovers all its sending neighbors to the destination are void area node, it will itself a void area node and retransmit backpressure beacon to its all neighbor node. All nodes won't send the data to the void area, in the event that they have other decision in their sending neighbor set. Through along these lines, when a void arise in the network, the void territory position message would be rapidly broadcast in the entire network. In the event that the packets achieve the void area node, the node will examine itself whether it is a void edge node. If it is not the $n$ it will send the packets to the sending void node whose ActualDelay is briefest until it accomplish a void edge node. At the point when packet achieve a void edge node, it will be conveyed by the void bypass scheme.

\section{- Void Bypass Scheme}

In FT-SPEED, the packets won't be dropped when they achieve the void edge nodes. The void edge nodes will attempt to sidestep the void and send the packets along the void edge till it find the node closer to the destination. However as they talked about in past Section, bypass the void need backtracking keeping in mind the end goal to achieve the destination. If backtracking, the nodes packets would have a negative transmission speed, which is not permitted by SPEED. Consequently in FT-SPEED, when the packets achieve the stuck node, it will no more take the speed necessity as the criteria to pick the following bounce node. It utilizes the known right hand rule talked about in GPSR to bypass the crevice, and just records every hop delay until the packets achieves the closer node. The time devoured in every hop is involved in every packet. The lost speed may be repaid when the packets achieve a closer node unless the deadline doesn't terminate during the bypassing.

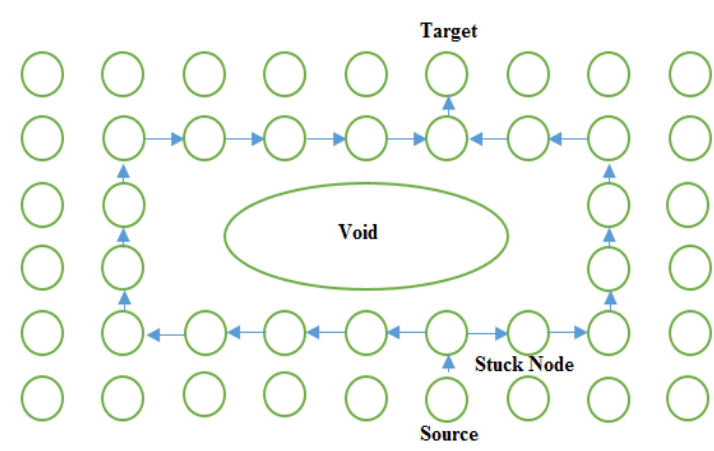

Fig 6: Example of Void Bypass

At the point when the first packet for target achieves the stuck node, a void area and ID step will begin. The received packet is sent to the accompanying void edge node by the acclaimed void discovering algorithm BOUNDHOLE [6] in the counterclockwise request. At the point when the packet reaches a closer node, it could be transmit with greedy forwarding technique again. Likewise, a copy of that packet will simply go along the limit of the void to perceive the void until it achieves the stuck node once more. At the point when the stuck node sees the past packet again by the parcel id, it will know the void could be associated by the void edge node. Additionally, every void edge node could record its preand successor void edge neighbors amid the packet passed on around the limit. At that point when void edge hub gets a packet not from the boundary node, it will sidestep the void on both sides to pass on the packet to the destination. Every nodes on the boundary will perform check the packet source when it gets a packet from the void edge node. On the off chance that the packet is from its preneighbor, it will send that packet to the edge neighbor. In the event the packet is send from the successor neighbor, then the packet will be sent to the preneighbor along the preedge. As a result of the flow of the sensor network, the void may develop or change shape amid the lifetime of the network. Subsequently, keeping up the fresh void information is additionally an enormous issue which ought to be taken care of. They acquaint the periodical support strategy to upgrade the void data. The stuck node will occasionally re-tries the void location and recognizable proof stride by send a control packet around the gap. At that point if there are any progressions about the void, all the edge node will know the difference about its edge neighbors soon. Supporting fault-tolerant communication in wsn is very important and challenging.

They introduce a fault-tolerant real-time protocol to upgrade the void issue in view of the work of SPEED. In FT-SPEED protocol, a novel void announce and bypass schemes are proposed. The packet could be swore from being sent to the void area by utilizing the void announce scheme. Void bypass scheme gives the sponsorship to course the packet around the both sides of the void, not just drop it. Nonetheless, in the event that they generally utilize the void edge nodes to convey the packets, the energy of the packet will be drained soon.

\subsection{EE-SPEED}

In this paper, they propose a methodology for SPEED protocol [7] to reduced residual energy in routing. Because of the constrained energy of a sensor node, energy efficient routing is a very vital issue in sensor networks. This approach discovers energy-efficient paths for delay constrained information in real-time traffic. The SPEED protocol do not consider energy metric in its coordinating. In the 
methodology, routing is in view of a weight function, which is a blend of the three variables: Speed, Energy and Delay. Here, the node with the highest esteem in the weight function is to be picked as the next hop forwarding. They extracting the network frequency by considering energy metric in routing decisions. This technique plans to build an about stateless routing protocol, which can be utilized to course information in the nodes residual energy. Simulation results exhibit that the new scheme enhances network lifetime around $15 \%$ more extended than the traditional SPEED protocol.

Answers are exuded by SPEED. Like other geographic routing algorithms, each node in SPEED intermittently transmit a beacon packet to its neighbors. This intermittent beaconing is only used for exchanging information (NeighborID, Position, SingleHopDelay, ExpireTime) between neighbors. Each node has a neighbor table, which comprises of a rundown of every one of its neighbors, where there is an entry for each neighbor. The Expire Time is utilized to timeout this entry in a neighbor table. The algorithm uses single hop delay as the metric to surmise the load of a node. Delay is calculated at the sender side, which timestamps the packet entering the network yield line and ascertains the round outing single hop deferral for this packet when tolerating the acknowledgement (ACK) at the receiver side, the length of time for transforming an ACK is put into the ACK packet. Propagation delay is overlooked. Each node has a Neighbor Set which is the situated of nodes that are inside the radio range $\mathrm{R}$ of node $\mathrm{i}$.

\subsubsection{Assumption}

Consider a network of $\mathrm{n}$ sensor nodes with none renew capable energy which are arbitrarily set in a network. They expect that the introductory energy dispersion on nodes is imbalanced, for the energy exhaustion rate is not the same on different nodes in the network. A few nodes have to expend more energy because of their location in the network. For instance, nodes closer to the base station are in a discriminating region, on the grounds that they need to forward data continuously, which brings about depleting their much quicker than the other nodes. The key configuration objective of this algorithm is to optimize energy utilization in the nodes, as they wish to prevent energy exhaustion on extraordinary nodes by adjusting the load on them. By this procedure they abstain partitioning of the network and expand its lifetime. Network lifetime can be characterized as the time it takes for the first node or a small amount nodes in the network to be drained of their energies. Since the lower energy nodes are what the network lifetime relies on, it gets to be important to offer them more assurance. The main point of interest of their way to deal with SPEED protocol is securing the nodes with less energy to stay away from their release and to have the nodes with more energy. Initial phase in the algorithm is to get the residual energy of the nodes. Next, they will form a weight function by which they could perform routing.

\subsubsection{Energy model}

Assume that there is an unbalanced distribution of starting energy on the nodes. Along these lines, the sink node and the neighboring ones have more beginning initial energy than the others. They demonstrate the residual energy on node $\mathrm{j}$ by $\mathrm{Ej}$, calculated based on the accompanying equation.

$$
E_{j}=\frac{E_{0}-\mathrm{C} E_{J}}{E_{0}}
$$

Where E0 is the initial energy and CEj is the expended energy of node $\mathrm{j}$, which can be ascertained in view of the model in. Energy utilization of every sensor is basically characterized as the normalized aggregate sum of energy spent in receiving or sending messages, i.e., CEj. Consequently, for every node, by having E0 value and computing $\mathrm{CEj}$, they could calculate its residue energy. As they had in SPEED protocol [3], each node intermittently broadcasts a beacon packet to its neighbors. The data passed by the beaconing is stored in a neighboring table. Every entry inside the table has the accompanying fields: (NeighborID, Position, SingleHopDelay, ExpireTime, and Residual Energy). Each node is location-aware. At that point, all nodes inside NSi set send their data to node i, and it spares them in FSi set, which utilizes this information to choose the following hop for its routing.

\subsubsection{Weight Function Definition and Calculation}

The present node i, considers its FSi set as the forwarding applicant. The forwarding applicant is looked over this situated,

$$
\begin{gathered}
\mathrm{f}=\max (\alpha \cdot \operatorname{En}+\beta \cdot \operatorname{Sp} \neq \emptyset(\mathrm{De})) \\
\text { Where } \alpha+\beta=1 \text { and } \\
\Phi(\mathrm{De})=\{1 \rightarrow D e>1 \text { Or } \\
=\{\mathrm{z} \rightarrow D e<1
\end{gathered}
$$

and the neighbor node with the most astounding value in the weight capacity has a higher probability to be picked as the forwarding node. This weight function is formally characterized as above. Here, fj is the weight function estimation of the jth sensor En is the proportion of residual energy on node $\mathrm{j}$, De is the proportion of delay on node $\mathrm{j}$, and $\mathrm{Sp}$ is the relay speed its packet exchanges from the present node to node j. $\alpha$ and $\beta$ are the coefficients of these elements. Having any of them lessened to zero, the relating segment will no more be considered. Presently, they characterize every element independently. So the residual energy is calculated as Here.

$$
E n_{j}=\frac{E_{J}}{E_{\max }}
$$

Where $\mathrm{Ej}$ is the estimation of residual energy on node $\mathrm{j}$, and Emax is the highest value of remaining residual energy on nodes in FSi set. The proportion of delay is

$$
D e_{j}=\frac{D_{J}}{d_{J}}
$$

Where $\mathrm{D}$ is the conceivable most extreme delay in a solitary bounce, (same as the delay threshold) and $\mathrm{dj}$ is the delay value on node $\mathrm{j}$ (singlehopdelay). The last parameter is ascertained in based on the accompanying formula

$$
S p_{j}=\frac{1-1 \_n e x t}{\text { singlehopdelay }}
$$

Where 1 is the separation from node $i$ to the destination, i next is the separation from the next hop forwarding candidate node to the destination, and delay is the evaluated delay to forward a packet to node $\mathrm{j}$ 
EE-SPEED is an algorithm for routing the information in the SPEED protocol that mainly focuses on the energy-efficient routing. In this routing algorithm, the thinking is to join the cost and metric the QoS metric on nodes to select the appropriate path by considering the three parameters of Speed, Energy and Delay. Simulation results and an examination of this calculation with the SPEED protocol represent to that dispersing energy utilization on nodes in routing will secure the nodes with less energy and keeps from a quick destruction. This, specifically, causes the network lifetime to increase.

\section{CONCLUSION AND FUTURE WORK}

From the protocols reviewed, though SPEED protocol, it has particular disadvantages. As its utilization the no state architecture therefore it consumes more energy and is has more void in the network. MMSPEED protocol tries to overcome the drawbacks of SPEED by using minimum overhead and multi path scheme. But it has more other deficiencies as same as the energy efficient and lower void avoidance. FT-SPEED overcomes these drawbacks by taking into consideration those factors and improves the performance in terms of void avoidance. It removes the congestion to the network and provide the path to packet to avoid voiding avoidance. The protocol EE-SPEED gives the method to provide more power efficient system. By these the MMSPEED, FT-SPEED and EE-SPEED is the extension and updatation of the SPEED Protocol which gives better performance, lower overhead, energy efficient and fault tolerance. Future works include the combination of FTSPEED and EE-SPEED to create a new protocol MEGASPEED. Also comparison and analysis with SPEED and MMSPEED routing protocols on the basis of QoS parameters such as Delay, throughput, Energy consumption and packet drop ratio. Improvisations on these parameters can also be performed to recuperate the end to end performance of SPEED protocol.

\section{REFERENCE}

[1] Akyildiz et al., "A Survey on Sensor Networks," IEEE Commun. Mag., vol. 40, no. 8, pp. 102-144, Aug. 2002.

[2] M. Fonoage, M. Cardei, and A. Ambrose," A QoS Based Routing Protocol for Wireless Sensor Networks," in IEEE 29th International Performance Computing and Communications Conference (IPCCC), pp.122 - 129, Albuquerque, NM, Dec. 2010.

[3] T. He, J. A. Stankovic, C. Lu, and T. F. Abdelzaher, "SPEED: A Stateless Protocol for Real-Time
Communication in Sensor Net-works," in 23rd International Conference on Distributed Computing Systems (ICDCS), pp. 46 - 55, May 2003.

[4] S. Felemban, E,Chang-Gun L.E..E,"MMSPEED QoS guarantee of reliability and. Timeliness in wireless sensor networks," Mobile Computing, IEEE Transactions, vol. 15, pp. 738 - 754, June 2006.

[5] L. Zhao, B. Kan, Y. Xu and X. Li1," FT-SPEED: A Fault Tolerant, Real time routing protocols for wireless sensor network," in International Conference on Wireless Communications, Networking and Mobile Computing (WiCom), pp. 2531 - 2534, Shanghai, Sept. 2007.

[6] Q.Fang, J.Gao and L.J. Guibas.” Locating and bypassing routing holes insensor networks". In Proc. Of IEEE Conference on Computer Communications (INFOCOM’04), Mar.2004.

[7] M. Sadegh, kordafshari, A. Pourkabirian, K. Faez, Ali M. Rahimabadi," Energy efficient SPEED routing protocol in wireless sensor network," in Fifth Advanced International Conference on Telecommunications (AICT), pp. 267 - 271, Venice, May 2009.

[8] M. Ibrahim Channa and Irum Memon," Real time traffic supports in wireless sensor network," in Student Conference on Engineering Sciences and Technology (SCONEST), pp. 1 - 6, Karachi, Aug 2005.

[9] T. He, J. A. Stankovic, C. Lu and T. F. Abdelzaher," A Spatiotemporal communication protocol for wireless sensor network," in IEEE Transactions on Parallel and Distributed Systems, vol. 16, pp. 995 - 1006, 2005.

[10] N. A. Pantazis, S. A. Nikolidakis and D. D. Vergados," Energy efficient routing protocols in wireless sensor network: A survey," in IEEE Communications Surveys \& Tutorials, vol.15, pp. 551-591, 2013.

[11] R. Roustaei, E. Zohrevandi, K. Hassani and A. Movaghar," A New Approach to Improve Quality of Service in SPEED Routing Protocol in Wireless Sensor Network through Data Aggregation," in Second International Conference on Environmental and Computer Science (ICECS), pp. 393 - 397, Dubai, Dec. 2009.

[12] Dargie W. and Poellabauer C., "Fundamentals of wireless sensor networks theory and practice," John Wiley and Sons, pp. 168-183, 191-192, 2010. 